\title{
ADDITIVE REPRESENTATION IN THIN SEQUENCES, II: THE BINARY GOLDBACH PROBLEM
}

\author{
J. BRÜDERN, K. KAWADA AND T. D. WOOLEY
}

$\S 1$. Introduction. Most prominent among the classical problems in additive number theory are those of Waring and Goldbach type. Although use of the Hardy-Littlewood method has brought admirable progress, the finer questions associated with such problems have yet to find satisfactory solutions. For example, while the ternary Goldbach problem was solved by Vinogradov as early as 1937 (see Vinogradov [16], [17]), the latter's methods permit one to establish merely that almost all even integers are the sum of two primes (see Chudakov [4], van der Corput [5] and Estermann [7]). Subsequent investigations have resulted in sharper estimates for the number of possible exceptions. In particular, writing $r_{1}(N)$ for the number of even natural numbers not exceeding $N$ which are not the sum of two primes, we have the celebrated theorem of Montgomery and Vaughan [10] which shows that ${ }_{1}(N) \ll N^{1-\delta}$ for some small $\delta>0$. One may seek to gain greater insight regarding the nature of any possible exceptional set by investigating the extent to which natural numbers in thinner sequences are represented in the proposed manner. The literature concerning such questions is presently enjoying a phase of rapid expansion, and now includes material on short intervals (see, for example, Ramachandra [13], Perelli and Pintz [12], Languasco and Perelli [9] and Baker, Harman and Pintz [1]), polynomial sequences (see Perelli [11]), and even rather sparse sequences provided by such sets as $\left\{\left[\exp \left((\log n)^{\gamma}\right)\right]: n \in \mathbb{N}\right\}$ for $1<\gamma<$ $3 / 2$ (see Brüdern and Perelli [3]). The goal of this paper is to provide methods for the binary Goldbach problem which address the problem of providing more refined information concerning exceptional sets in polynomial sequences.

In order to set the scene for the specific problem at hand, we recall a result of Perelli [11]. Let $\Phi \in \mathbb{Z}[x]$ be a polynomial of degree $k$ with positive leading coefficient, and let ${ }_{k}(N ; \Phi)$ denote the number of natural numbers $n$, with $1 \leqslant n \leqslant N$, for which the equation

$$
2 \Phi(n)=p_{1}+p_{2}
$$

has no solution in primes $p_{1}, p_{2}$. Then Perelli shows that, for any positive number $A$, one has

$$
{ }_{k}(N ; \Phi) \ll_{A, \Phi} N(\log N)^{-A},
$$

whence almost all values of the polynomial $2 \Phi(n)$ are the sum of two primes. We are able to improve considerably the quality of the estimate (1). Thus, in $\S 4$, we establish the following theorem.

THEOREM 1. Let $\Phi \in \mathbb{Z}[x]$ be a polynomial of degree $k$ with positive leading coefficient, and let ${ }_{k}(N ; \Phi)$ be as defined in the previous paragraph. Then there 
is an absolute constant $c>0$ such that

$$
\epsilon_{k}(N ; \Phi) \ll_{\Phi} N^{1-c / k}
$$

We remark that estimates of the strength provided by Theorem 1 are not even available in the literature under the assumption of the Riemann Hypothesis for Dirichlet $L$-functions (see Perelli [11]). With little additional effort it is possible to establish a significantly stronger conclusion than that of Theorem 1 in the case that $\Phi(t)=t^{k}$, provided that one is prepared to restrict attention to smooth values of $t$. Let

$$
\not(P, R)=\{n \in[1, P] \cap \mathbb{Z}: p \text { prime, } p \mid n \Rightarrow p \leqslant R\} .
$$

Also, let $\tilde{f}_{k}(N, R)$ denote the number of natural numbers $n \in,(N, R)$, for which the equation $2 n^{k}=p_{1}+p_{2}$ has no solution in primes $p_{1}, p_{2}$.

THEOREM 2. There exist positive absolute constants $\eta_{0}$ and $\theta$ such that, for every natural number $k$, whenever $0<\eta<\eta_{0}$, and $R$ is a real number with $N^{\eta / 2}<R \leqslant N^{\eta}$, then one has

$$
\tilde{\tau}_{k}(N, R) \ll_{k, \eta} N^{1-\theta} .
$$

We refer the reader to our earlier paper [2] for a lengthy discussion concerning the broad ideas and philosophy underlying our approach to estimating exceptional sets in thin sequences. For now, suffice it to say that we consider the possible set of exceptions in the binary Goldbach problem directly, employing an exponential sum over the latter exceptions, and exploiting mean values of this sum within our application of the Hardy-Littlewood method. It is crucial to our argument that this exponential sum preserve arithmetic information concerning the set of exceptions, information which is lost, or at least exploited rather inefficiently, in more traditional approaches involving the use of Bessel's inequality.

Throughout, the letter $\varepsilon$ will denote a sufficiently small positive number. We take $N$ to be the basic parameter, a large real number depending at most on $k, \varepsilon$, and any coefficients of implicit polynomials if necessary. We use « and $\gg$ to denote Vinogradov's well-known notation, implicit constants depending at most on $k, \varepsilon$ and implicit polynomials. Also, we write $[x]$ for the greatest integer not exceeding $x$. Summations start at 1 unless indicated otherwise. In an effort to simplify our analysis, we adopt the following convention concerning the parameter $\varepsilon$. Whenever $\varepsilon$ appears in a statement, we assert that, for each $\varepsilon>0$, the statement holds for sufficiently large values of the main parameter. Note that the "value" of $\varepsilon$ may consequently change from statement to statement, and hence also the dependence of implicit constants on $\varepsilon$.

§2. An averaged minor arc contribution. A little preparation is required before embarking on our quest for Theorem 1 in earnest. Let $\Phi \in \mathbb{Z}[t]$ be a polynomial of degree $k$ with positive leading coefficient. It is convenient to 
write $\kappa=2^{-1 / k}$. Let ${ }_{k}^{*}(N ; \Phi)$ denote the number of natural numbers $n$, with $\kappa N<n \leqslant N$, for which the equation

$$
2 \Phi(n)=p_{1}+p_{2}
$$

has no solution in primes $p_{1}, p_{2}$. We aim to show that there is an absolute constant $c>0$ such that ${ }_{k}^{*}(N ; \Phi) \ll N^{1-c / k}$. The conclusion of Theorem 1 follows by collecting together the exceptional $n$ from intervals $\kappa^{\prime} N<n \leqslant \kappa^{l-1} N$, where $l \in \mathbb{N}$.

Let $N$ be a large real number, and write $X=2 \Phi(N)$. Then plainly one has $X \asymp N^{k}$. We take $\delta$ to be a sufficiently small, though fixed, positive real number to be chosen later, and write $P=X^{6 \delta}$. We define the exponential sum $S(\alpha)$ by

$$
S(\alpha)=\sum_{p<p \leqslant X}(\log p) e(\alpha p),
$$

where the summation is over prime numbers, and when $\mathfrak{B} \subseteq[0,1]$ we write

$$
r(n ; \mathfrak{B})=\int_{\mathfrak{Z}} S(\alpha)^{2} e(-\alpha n) d \alpha .
$$

For brevity we write $r(n)=r(n ;[0,1])$, and note that $r(2 \Phi(n))$ counts the solutions of (2) with weight $\left(\log p_{1}\right)\left(\log p_{2}\right)$. We apply the Hardy-Littlewood method, defining the major arcs $\mathfrak{M}$ to be the union of the intervals

$$
\mathfrak{M}(q, a)=\left\{\alpha \in[0,1]:|q \alpha-a| \leqslant P X^{-1}\right\}
$$

with $0 \leqslant a \leqslant q \leqslant P$ and $(a, q)=1$. We define the minor arcs $m$ by $\mathfrak{m}=[0,1] \backslash \mathfrak{M}$, and note that, for each $n$ with $\kappa N<n \leqslant N$, one has

$$
r(2 \Phi(n))=r(2 \Phi(n) ; \mathfrak{M})+r(2 \Phi(n) ; \mathfrak{m}) .
$$

We first show that, on average, the contribution of the minor arcs in (4) is small. It is here that we profit handsomely compared to previous treatments.

Lemma 1. There is a positive real number $a=a(\delta)$, depending at most on $\delta$, such that

$$
\sum_{\kappa N<n \leqslant N}|r(2 \Phi(n) ; \mathfrak{m})| \ll X N^{1-a / k} .
$$

Proof. Motivated by the observation that the expression $r(l ; \mathfrak{m})$ is real for every natural number $l$, we define $\eta(l)$, for each $l \in \mathbb{N}$, by

$$
\eta(l)=\left\{\begin{aligned}
1, & \text { when } r(l ; \mathfrak{m}) \geqslant 0, \\
-1, & \text { when } r(l ; \mathfrak{m})<0 .
\end{aligned}\right.
$$

Thus it follows from (3) that

$$
\sum_{\kappa N<n \leqslant N}|r(2 \Phi(n) ; \mathrm{m})|=\int_{\mathfrak{m} \mathfrak{l}} S(\alpha)^{2} K(-\alpha) d \alpha,
$$

where 


$$
K(\alpha)=\sum_{\kappa N<n \leqslant N} \eta(2 \Phi(n)) e(2 \Phi(n) \alpha) .
$$

We next observe that, when $t$ is an even natural number, then the mean value $\int_{0}^{1}|K(\alpha)|^{t} d \alpha$ counts the number of solutions of an underlying diophantine equation, with each solution counted with weight at most 1 in modulus, whence it follows that

$$
\int_{0}^{1}|K(\alpha)|^{t} d \alpha \leqslant \int_{0}^{1}\left|\sum_{n \leqslant N} e(2 \Phi(n) \alpha)\right|^{t} d \alpha .
$$

Let

$$
t=2[(2-\log \delta)] k^{2}
$$

Then, by combining a classical version of Vinogradov's mean value theorem (see, for example, Vaughan [15], Theorem 5.1) with Theorem 1 of Ford [8], one obtains, for each integer $m$ with $1 \leqslant m \leqslant k$, the estimate

$$
\int_{0}^{1}|K(\alpha)|^{t} d \alpha \ll N^{t-k+\Delta / m},
$$

where

$$
\Delta=\frac{1}{2} k^{2} \exp \left(-(t-2 k-m(m-1)) /\left(2 k^{2}\right)\right)<\delta k^{2} .
$$

We take $m=k$, and thereby deduce that

$$
\int_{0}^{1}|K(\alpha)|^{t} d \alpha \ll N^{t-(1-\delta) k}
$$

Finally, we note that by orthogonality one has

$$
\int_{0}^{1}|S(\alpha)|^{2} d \alpha=\sum_{P<p \leqslant X}(\log p)^{2} \ll X \log X,
$$

and that, by Vaughan [15], Theorem 3.1,

$$
\sup _{\alpha \in \mathfrak{m}}|S(\alpha)| \ll X P^{-1 / 2}(\log X)^{4} \ll X^{1-3 \delta}(\log X)^{4} .
$$

On applying Hölder's inequality to (5), one obtains

$$
\begin{aligned}
& \sum_{\kappa N<n \leqslant N}|r(2 \Phi(n) ; \mathrm{m})| \\
& \quad \leqslant\left(\sup _{\alpha \in \mathfrak{m}}|S(\alpha)|\right)^{2 / t}\left(\int_{0}^{1}|S(\alpha)|^{2} d \alpha\right)^{1-1 / t}\left(\int_{0}^{1}|K(\alpha)|^{t} d \alpha\right)^{1 / t}
\end{aligned}
$$


whence, on recalling that $X \asymp N^{k}$ and making use of (7)-(9), we conclude from (6) that

$$
\begin{aligned}
\sum_{\kappa N<n \leqslant N}|r(2 \Phi(n) ; \mathfrak{m})| & \ll N^{1-k(1-\delta) / t}(X \log X)^{1-1 / t} X^{(2-6 \delta) / t}(\log X)^{8 / t} \\
& \ll N X^{1-5 \delta / t}(\log X)^{3},
\end{aligned}
$$

and the desired conclusion follows immediately.

\$3. The major arc contribution. Our treatment of the major arc contribution in (4) depends heavily on the work of Montgomery and Vaughan [10], and in order to make use of the latter we will require some additional notation. Recall that there exists a positive constant $c_{1}>0$ such that

$$
L(\sigma, \chi) \neq 0 \quad \text { for } \sigma \geqslant 1-\frac{c_{1}}{\log P},
$$

for all primitive Dirichlet characters $\chi$ of modulus $q \leqslant P$, with the possible exception of at most one real primitive character, henceforth called the exceptional character. If such a character exists, we write $\tilde{\chi}$ for this exceptional character, and $\tilde{r}$ for its modulus. It then follows that $L(s, \tilde{\chi})$ has a unique zero $\tilde{\beta}$ violating (10), and moreover one has

$$
\tilde{r}^{-1 / 2}(\log \tilde{r})^{-2} \ll 1-\tilde{\beta} \leqslant \frac{c_{1}}{\log P},
$$

in which the implicit constant is absolute. We refer the reader to Davenport [6], Chapter 14, for an account of such matters.

Lemma 2. Suppose that $Y$ is a real number with $1 \leqslant Y \leqslant X^{\delta / k}$. Then one has

$$
r(2 \Phi(n) ; \mathfrak{M}) \gg X Y^{-1 / 2}(\log X)^{-1}
$$

for all $n$ satisfying $\kappa N<n \leqslant N$, with the possible exception of $O\left(N^{1+\varepsilon} Y^{-1}\right)$ values of $n$.

Proof. For the proof of this lemma, we follow closely $\S 8$ of Montgomery and Vaughan [10]. In this context, it may be useful to the reader to note that the expression $R_{1}(n)$ in the latter corresponds to $r(n ; \mathfrak{M})$ in the present paper.

Suppose first that there is no exceptional character. Then, as in $\S 8$ of Montgomery and Vaughan [10], it follows that for $\kappa N<n \leqslant N$ one has

$$
r(2 \Phi(n) ; \mathfrak{M}) \gg X,
$$

and the conclusion of the lemma is immediate. Suppose then that the exceptional character exists. Then again we find from Montgomery and Vaughan [10], $\S 8$, that, when $\kappa N<n \leqslant N$ and $(2 \Phi(n), \tilde{r})=1$, one has the lower bound (12). We therefore deduce that the lemma will follow, on showing that the number of integers $n$ satisfying $\kappa N<n \leqslant N$, for which

$$
(2 \Phi(n), \tilde{r})>1 \quad \text { and } \quad r(2 \Phi(n) ; \mathfrak{M}) \ll X Y^{-1 / 2}(\log X)^{-1},
$$

is at most $O\left(N^{1+\varepsilon} Y^{-1}\right)$. 
Observe next that, when $(2 \Phi(n), \tilde{r})>1$, one has $\tilde{\chi}(2 \Phi(n))=0$. Under such circumstances, it follows from equations (6.17) and (7.1) of Montgomery and Vaughan [10] that

$$
|r(2 \Phi(n) ; \mathfrak{M})-2 \Phi(n) \Xi(2 \Phi(n))-\tilde{I}(2 \Phi(n)) \tilde{\Xi}(2 \Phi(n))| \leqslant c_{2}\left(T_{1}+T_{2}\right),
$$

where $c_{2}$ is a positive absolute constant,

$$
T_{1}=X^{1+\delta} P^{-1}(2 \Phi(n), \tilde{r}), \quad T_{2}=\frac{2 \Phi(n)}{\varphi(2 \Phi(n))}(1-\tilde{\beta}) X e^{-c_{3} / \delta} \log P
$$

and here again $c_{3}$ is a positive absolute constant, the function $\varphi$ denotes the Euler totient, and for the purposes of the present discussion it suffices to note that, by (6.16), (6.18), (8.5) and the argument following (6.17) of Montgomery and Vaughan [10], one has, for each $n$ with $\kappa N<n \leqslant N$,

$$
\begin{aligned}
\Xi(2 \Phi(n)) & \geqslant \frac{\Phi(n)}{\varphi(2 \Phi(n))} \geqslant 1, \\
\tilde{I}(2 \Phi(n)) & \leqslant(2 \Phi(n))^{\tilde{\beta}} \leqslant 2 \Phi(n), \\
|\tilde{S}(2 \Phi(n))| & \leqslant \Xi(2 \Phi(n)) \prod_{\substack{p \mid \tilde{r} \\
p i \sigma(n)}}(p-2)^{-1} .
\end{aligned}
$$

We note further that, since $\tilde{r}$ is the modulus of a real primitive character, then necessarily $\tilde{r}$ takes the shape

$$
\tilde{r}=2^{v} u \text {, }
$$

where $v$ and $u$ are integers with $0 \leqslant v \leqslant 3,2 \nmid u$ and $\mu(u)^{2}=1$.

Since $\tilde{r}$ takes the shape (18) with $u$ square-free, the integers $n$ with $\kappa N<n \leqslant N$ and $(2 \Phi(n), \tilde{r})>Y$ number at most

$$
\sum_{\substack{d \mid r \\ d>Y}} \sum_{\substack{n<N \\ d>2 \Phi(n)}} 1 \ll \sum_{\substack{d \mid r \\ d>Y}} d^{\varepsilon}(1+N / d) \ll N^{1+\varepsilon} Y^{-1}
$$

In consequence, we may discard these integers without loss, counting them amongst the possible exceptions of the statement of the lemma. Suppose then that $n$ is an integer with $\kappa N<n \leqslant N$ and $1<(2 \Phi(n), \tilde{r}) \leqslant Y$. Then one finds from (14) that

$$
T_{1} \leqslant X^{1-\delta} Y^{-1 / 2} .
$$

Further, if the product in (17) is non-empty, then it follows from (13)-(17), (19) and (11) that, when $\delta$ is sufficiently small in terms of $c_{1}, c_{2}, c_{3}$, one has

$$
r(2 \Phi(n) ; \mathfrak{M}) \geqslant\left(1-9 c_{1} c_{2} e^{-c_{3} / \delta}\right) \subseteq(2 \Phi(n)) \Phi(n) \gg X,
$$

and the bound (12) again holds.

Suppose then that the product in (17) is empty, whence by (18) one has

$$
(2 \Phi(n), \tilde{r}) \geqslant \prod_{\substack{p \mid r \\ p>3}} p \geqslant \frac{\tilde{r}}{24} .
$$


Since by hypothesis we have $(2 \Phi(n), \tilde{r}) \leqslant Y$, it follows that $\tilde{r} \ll Y$. We therefore deduce from (16), together with (6.21) of Montgomery and Vaughan [10], that for each such $n$ with $\kappa N<n \leqslant N$ one has

$$
2 \Phi(n) \circlearrowleft(2 \Phi(n))+\tilde{I}(2 \Phi(n)) \Xi(2 \Phi(n)) \geqslant c_{4} \Phi(n) \circlearrowleft(2 \Phi(n))(1-\tilde{\beta}) \log P
$$

for a certain absolute positive constant $c_{4}$. For this last class of integers $n$, therefore, it follows from (13), (14) and (19) that

$$
\begin{aligned}
& r(2 \Phi(n) ; \mathfrak{M}) \\
& \geqslant\left(c_{4}-9 c_{2} e^{-c_{3} / \delta}\right) \circlearrowleft(2 \Phi(n)) \Phi(n)(1-\tilde{\beta}) \log P+O\left(X^{1-\delta} Y^{-1 / 2}\right)
\end{aligned}
$$

whence, by (11), (15) and our earlier observation that $\tilde{r} \ll Y$, we conclude that, whenever $\delta$ is sufficiently small in terms of $c_{1}, c_{2}, c_{3}, c_{4}$, one has

$$
r(2 \Phi(n) ; \mathfrak{M}) \gg X Y^{-1 / 2}(\log X)^{-1} \text {. }
$$

Collecting together our earlier conclusions, we find that the lower bound (20) holds for all integers $n$ satisfying $\kappa N<n \leqslant N$, with at most $O\left(N^{1+\varepsilon} Y^{-1}\right)$ possible exceptions. This completes the proof of the lemma.

\$4. The proofs of Theorems 1 and 2. The proof of Theorem 1 may now be swiftly overwhelmed. We take $\delta$ to be a fixed positive number, sufficiently small in the context of Lemmas 1 and 2 , and write $\tau=\frac{1}{2} \min \{\delta, a(\delta)\}$. We then take $Y=N^{\tau / k}$, and note that by Lemma 1 we have

$$
r(2 \Phi(n) ; \mathfrak{m}) \ll X Y^{-1}
$$

for each integer $n$ satisfying $\kappa N<n \leqslant N$, with at most $E_{1}$ possible exceptions, where $E_{1}$ satisfies

$$
E_{1} \ll \frac{Y}{X} \sum_{\kappa N<n \leqslant N}|r(2 \Phi(n) ; \mathrm{m})| \ll Y N^{1-a / k} \ll N Y^{-1} .
$$

On the other hand, it follows from Lemma 2 that (20) holds for all integers $n$ with $\kappa N<n \leqslant N$, with at most $O\left(N^{1+\varepsilon} Y^{-1}\right)$ possible exceptions. On recalling (4), we find that $r(2 \Phi(n))>0$ for each integer $n$ with $\kappa N<n \leqslant N$, with at most $O\left(N^{1-\tau / k+\varepsilon}\right)$ possible exceptions. The conclusion of Theorem 1 is now immediate.

So far as the proof of Theorem 2 is concerned, we note merely that the methods of Wooley [18] (see the corollary to Wooley [19], Theorem 2.1; the methods of Vaughan [14] would also suffice for our purpose) show that, with $t=2[(2-\log \delta)] k$, one has for sufficiently small $\eta$ the bound

$$
\int_{0}^{1}\left|\sum_{\substack{x \in n \leqslant N \\ n \in(N, R)}} e\left(\alpha n^{k}\right)\right|^{t} d \alpha \ll_{\eta} N^{l-k+\Delta}
$$

where $\Delta=k \exp (1-t / k)<\delta k$. Then the argument of the proof of Lemma 1 shows that, for some positive real number $a=a(\delta) \leqslant \delta$, 


$$
\sum_{\substack{\kappa N<n \leqslant N \\ n \in .}}\left|\int_{\mathfrak{M}} S(\alpha)^{2} e\left(-2 n^{k} \alpha\right) d \alpha\right| \ll_{\eta} X N^{1-a},
$$

and here again we use the definitions introduced in $\$ 2$ of this paper. But Lemma 2 shows that

$$
\int_{\mathfrak{P}_{i}} S(\alpha)^{2} e\left(-2 n^{k} \alpha\right) d \alpha \gg X N^{-a / 3}
$$

for all $n$ with $\kappa N<n \leqslant N$ and $n \in \mathscr{W}(N, R)$, with the exception of at most $O\left(N^{1-a / 2}\right)$ values of $n$. Then, on recalling that our hypotheses concerning $R$ ensure that card $(K(N, R) \cap[\kappa N, N]) \gg_{\eta} N$, we may apply the argument used to conclude the proof of Theorem 1 to deduce that, for each integer $n$ satisfying $n \in \mathscr{Q}(N, R) \cap[\kappa N, N]$, with at most $O\left(N^{1-a / 10}\right)$ possible exceptions, one has $r\left(2 n^{k}\right)>0$. When $\eta_{0}$ is sufficiently small but positive, the conclusion of Theorem 2 is immediate.

Acknowledgements. The third author is a Packard fellow, and is supported in part by NSF grant DMS-9622773. This paper benefitted from visits of various of the authors to Ann Arbor, Kyoto, Oberwolfach and Stuttgart, and the authors collectively thank these institutions for their hospitality and excellent working conditions.

\section{References}

1. R. C. Baker, G. Harman and J. Pintz. The exceptional set for Goldbach's problem in short intervals. Sieve Methods Exponential Sums, and their Applications in Number Theory (Cardiff 1995) (G. Greaves, G. Harman and M. Huxley, eds.), (Cambridge University Press, Cambridge, 1997), pp. 1-54.

2. J. Brüdern, K. Kawada and T. D. Wooley. Additive representation in thin sequences, I: Waring's problem for cubes. Ann. Sci. Ecole Norm. Sup. (4), 34 (2001), 471-501.

3. J. Brüdern and A. Perelli. Goldbach numbers in sparse sequences. Ann. Inst. Fourier, Grenoble, 48 (1998), 353-378.

4. N. G. Chudakov. On the density of the set of even numbers which are not representable as a sum of two odd primes. Izv. Akad. Nauk SSSR Ser. Mat., 2 (1938), 25-40.

5. J. G. van der Corput, Sur I'hypothèse de Goldbach. Proc. Akad. Wet. Amsterdam, 41 (1938), 76-80.

6. H. Davenport. Multiplicative Number Theory (2nd edn.). (Springer-Verlag, Berlin-New York, 1980).

7. T. Estermann. On Goldbach's problem: Proof that almost all even positive integers are sums of two primes. Proc. London Math. Soc. (2), 44 (1938), 307-314.

8. K. B. Ford. New estimates for mean values of Weyl sums. Internat. Math. Res. Notices (1995), $155-171$.

9. A. Languasco and A. Perelli. A pair correlation hypothesis and the exceptional set in Goldbach's problem. Mathematika, 43 (1996), 349-361.

10. H. L. Montgomery and R. C. Vaughan. The exceptional set in Goldbach's problem. Acta Arith., 27 (1975), 353-370.

11. A. Perelli. Goldbach numbers represented by polynomials. Rev. Mat. Iheroamericana, 12 (1996), 477-490.

12. A. Perelli and J. Pintz. On the exceptional set for Goldbach's problem in short intervals. $J$. London Math. Soc. (2), 47 (1993), 41-49.

13. K. Ramachandra. On the number of Goldbach numbers in small intervals. J. Indian Math. Soc., 37 (1973), 157-170.

14. R. C. Vaughan. A new iterative method in Waring's problem. Acta Math., 162 (1989), 1-71. 
15. R. C. Vaughan. The Hardy-Littlewood Method (2nd edn.). (Cambridge University Press, Cambridge, 1997).

16. I. M. Vinogradov. Representation of an odd integer as a sum of three primes. C. R. Acad. Sci. URSS, 15 (1937), 6-7.

17. I. M. Vinogradov. Some theorems concerning the theory of primes. Mat. Sbornik, 2 (1937), $179-195$.

18. T. D. Wooley, Large improvements in Waring's problem. Ann. Math. (2), 135 (1992), 131164.

19. T. D. Wooley. The application of a new mean value theorem to the fractional parts of polynomials. Acta Arith., 65 (1993), 163-179.

Professor J. Brüdern,

Mathematisches Institut A,

Universität Stuttgart,

Postfach 801140 ,

D-70511 Stuttgart,

Germany.

E-mail: bruedern $(\omega)$ mathematik.uni-stuttgart.de

Professor K. Kawada,

Department of Mathematics,

Faculty of Education,

Iwate University,

Morioka 020-8550,

Japan.

E-mail: kawada@iwate-u.ac.jp

Professor T. D. Wooley,

Department of Mathematics,

University of Michigan,

East Hall,

525 East University Avenue,

Ann Arbor, MI 48109-1109,

U.S.A.

E-mail: wooley@math.lsa.umich.edu
11P32: NUMBER THEORY; Additive number theory; partitions; Goldbach-type theorems; other additive questions involving primes.
Received on the 20th of March, 2000. 